\title{
Imaging spectrum of peripheral primitive neuroendocrine tumours
}

Pankaj Gupta ${ }^{1}$, MD, Smriti $\underline{\text { Hari }}^{1}$, MD, Sanjay $\underline{\text { Thulkar }}^{1}$, MD

ABSTRACT Primitive neuroectodermal tumours (PNETs) arise from pluripotent neural crest cells and are classified as either central or peripheral. Peripheral PNETs (pPNETs) arise outside the central nervous system and sympathetic chain. These rare neoplasms comprise only $1 \%$ of all sarcomas and have highly aggressive biological behaviour and dismal prognosis. Adolescents and young adults are typically affected. Only isolated case reports on pPNETs appearing in both typical and atypical sites can be found in the literature. Timely diagnosis of pPNETs is a challenge to clinicians and radiologists due to the disease's insidious onset and variable locations, coupled with the limited studies that focus on the imaging features of pPNETs. Hence, this article serves to review the imaging features of this rare tumour.

Keywords: Ewing's sarcoma, peripheral primitive neuroectodermal tumour, primitive neuroectodermal tumour, sarcomas

\section{INTRODUCTION}

Primitive neuroectodermal tumours (PNETs) are highly aggressive neoplasms that are classified as either central or peripheral. Peripheral PNETs (pPNETs) are a rare group of malignant small round cell tumours that arise from pluripotent neural crest cells, outside the central and sympathetic nervous system. pPNETs differ from central PNETs in that they typically express high amounts of the MIC2 antigen (CD 99) and have highly characteristic chromosomal translocation - features also shared by Ewing's sarcoma (ES).(1) Thus, pPNETs and skeletal ES belong to the same family, with 'ES/PNET' now being the preferred term for this condition. ${ }^{(2)}$ The presence of neural differentiation in PNETs distinguishes it from ES. ${ }^{(3)}$ The common features shared by the two tumours include small round cell morphology, immunohistochemistry (positive for O13, a monoclonal antibody for the p30/32MIC2 gene product) and chromosomal translocation involving the ES gene $[t(11 ; 22)(q 24 ; q 12)] .{ }^{(4)}$ Neural differentiation in pPNETs can be demonstrated using immunohistochemistry or electron microscopy. The immunohistochemical markers of neural differentiation include S-100 protein, neuron-specific enolase, leucine 7 , chromogranin $A$, synaptophysin, protein gene product 9.5, secretogranin II, vimentin and keratin. ${ }^{(5)}$ Distinct electron microscopic features include neurosecretory granules, neurofilaments and neurotubules. ${ }^{(6)}$

Although it pPNET can affect persons of any age group, the peak incidence is during adolescence and young adulthood (median age 17 years). ${ }^{(7)}$ A slight male predilection has been reported in some studies. ${ }^{(8)}$ The clinical presentation varies depending on the site of involvement. In general, the disease has an insidious onset, aggressive course, large size of the primary mass, and metastases to the lungs or bones in onethird of patients. ${ }^{(8)}$ The chest wall is the single most common site of involvement, and PNETs located in this site are known as Askin tumours. ${ }^{(2)}$ Other common sites of involvement are the thoracopulmonary region, the intra-abdominal, intrapelvic and retroperitoneal soft tissues, the head and neck regions, and the extremities. ${ }^{(3)}$

Regardless of the site of involvement, pPNETs share certain imaging features, which include a large soft tissue component with frequent central necrosis and moderate heterogeneous enhancement after intravenous contrast administration. Bone involvement manifests as permeative lytic destruction, although there can be a component of sclerosis, leading to a mixed pattern. In general, the soft tissue component tends to displace, rather than encase, the adjacent organs; however, extension along the neural foramina is commonly noted in retroperitoneal or paraspinal PNETs. ${ }^{(9)}$ Vascular involvement is common and presents as contour abnormality, tumour-induced stenosis or occlusion. Calcification (either coarse or fine) and haemorrhage are uncommon at presentation. Regional lymphadenopathy is also infrequently detected. ${ }^{(10)}$ Most tumours have isointense to slightly hyperintense $\mathrm{T} 1$-weighted signal and heterogeneous hyperintense T2-weighted signal, compared to the adjacent normal muscle. These signal characteristics indicate the heterogeneity of the tumour, with areas of necrosis and haemorrhage. Positron emission tomography plays an important role in the detection of metastatic disease.

Treatment involves a multimodality approach with extensive surgical resection, multi-agent chemotherapy and radiotherapy. However, despite extensive treatment, the overall prognosis remains dismal for this highly aggressive malignancy.

${ }^{1}$ Department of Radiology, All India Institute of Medical Sciences, New Delhi, India

Correspondence: Dr Pankaj Gupta, Resident, Department of Radiology, All India Institute of Medical Sciences, New Delhi 110029, India. pankajgupta959@gmail.com 

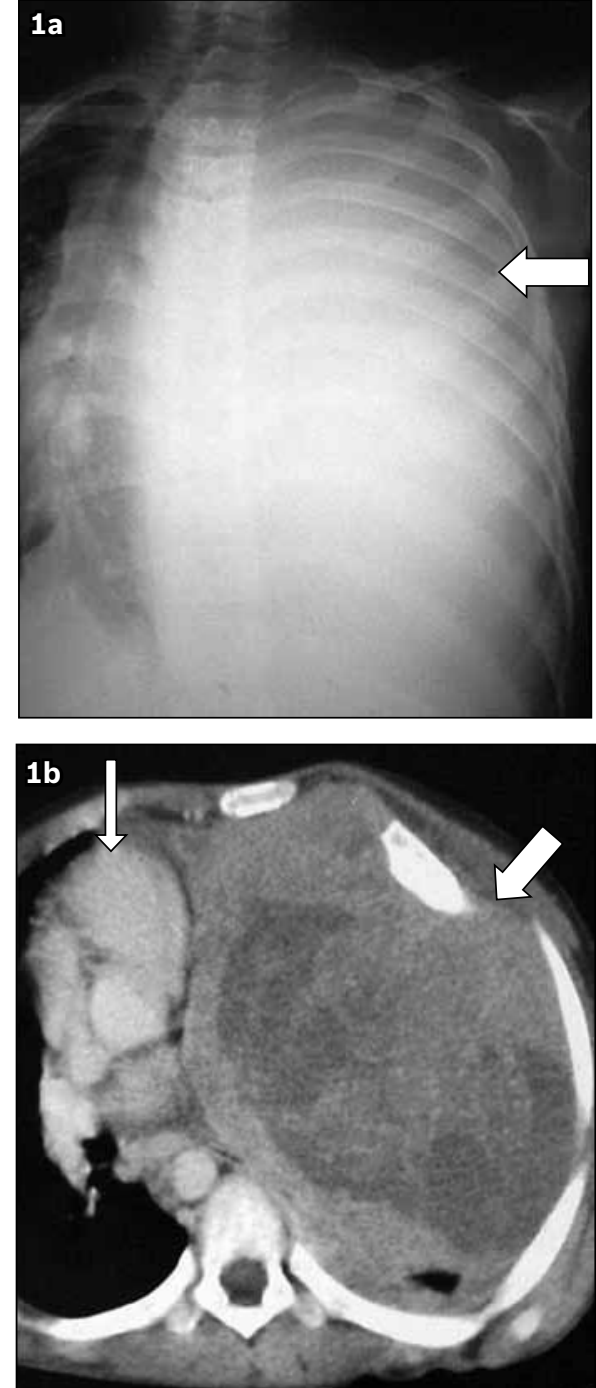

Fig. 1 Chest wall PNET (Askin tumour). (a) Chest radiograph of a 10 -year-old boy shows an opaque left hemithorax (arrow) with contralateral mediastinal shift and mass effect on the right lung; no rib destruction is evident. (b) Contrast-enhanced CT image of the same patient shows a large heterogeneous mass (thick arrow) occupying the left chest, displacing the heart and aorta (thin arrow) toward the right; note the lack of encasement or invasion of these structures.

\section{SPECIFIC SITES}

\section{Chest wall (Askin tumour)}

The chest wall is the most common site of pPNETs. Patients typically present with chest pain and swelling, as well as and respiratory distress. It usually occurs as a solitary mass lesion centred on a rib that shows a permeative lytic or mixed lytic sclerotic pattern of destruction. The mass may be huge. A common radiographic presentation is unilateral opaque hemithorax (Fig. 1a). ${ }^{(6)}$ Rib destruction and soft tissue swelling can also be visualised on plain chest radiographs. Pleural effusion is not a common feature. Large lesions demonstrate components of the mass in intrathoracic, intraspinal and retroperitoneal compartments (Figs. 1b \& 2). Computed tomography $(\mathrm{CT})$ and magnetic resonance (MR) imaging features follow the pattern previously described. In addition, MR imaging can detect the extent of chest wall invasion.

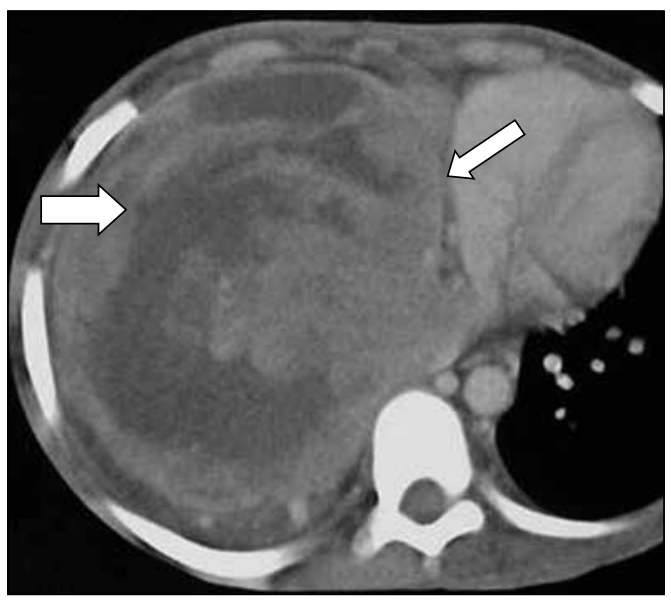

Fig. 2 Chest wall PNET (Askin tumour). Contrast-enhanced chest CT image of a 10-year-old boy shows a large mass occupying the right hemithorax (thick arrow). There are large nonenhancing areas within the mass, suggestive of necrosis. Note that the mass respects the fat planes (thin arrow) and pushes the heart and vessels toward the left, without any tendency to invade or encase.

The suspicion of perilymphatic and perineural extension of the tumour can be raised if nodular or linear subpleural densities are noticed on CT. ${ }^{(6)}$ Differential diagnoses include neuroblastoma metastases, rhabdomyosarcoma and lymphoma. Features that are helpful for differentiating the aforementioned entities from PNET include age at presentation (neuroblastoma metastases typically present in the early part of the first decade, whereas rhabdomyosarcomas typically occur in the latter half of the first decade) and the absence of regional lymphadenopathy (common in lymphoma).

\section{Abdomen and pelvis}

The retroperitoneum is one of the common sites of involvement for pPNETs. They can arise from the pelvic bones (Fig. 3), presacral soft tissue, kidneys, adrenals, and perirenal or periureteric spaces. ${ }^{(11)}$ Masses located in these sites tend to be large, with features similar to PNETs occurring elsewhere.

\section{Extremity}

Another common location of pPNETs is the long bones. Lesions commonly involve the humerus (Fig. 4), femur (Fig. 5a) and tibia (Fig. 5b). Uncommon sites such as the calcaneum (Fig. 6) are also encountered. Lesions are typically permeative lytic in nature. Sclerosis is a finding in lesions that are healing. An associated soft tissue mass can vary in size and follows the features noted for PNETs at other locations. Subperiosteal spread of the tumour has been described in some studies. ${ }^{(9)}$

\section{Scapula}

PNETs of the scapula are uncommon. Clinically, patients present with back pain and swelling. On imaging, these pPNETs appear as large soft tissue masses with associated erosion of the scapula (Fig. 7). PNETs located at this site are noted to have a better prognosis. ${ }^{(10)}$ 

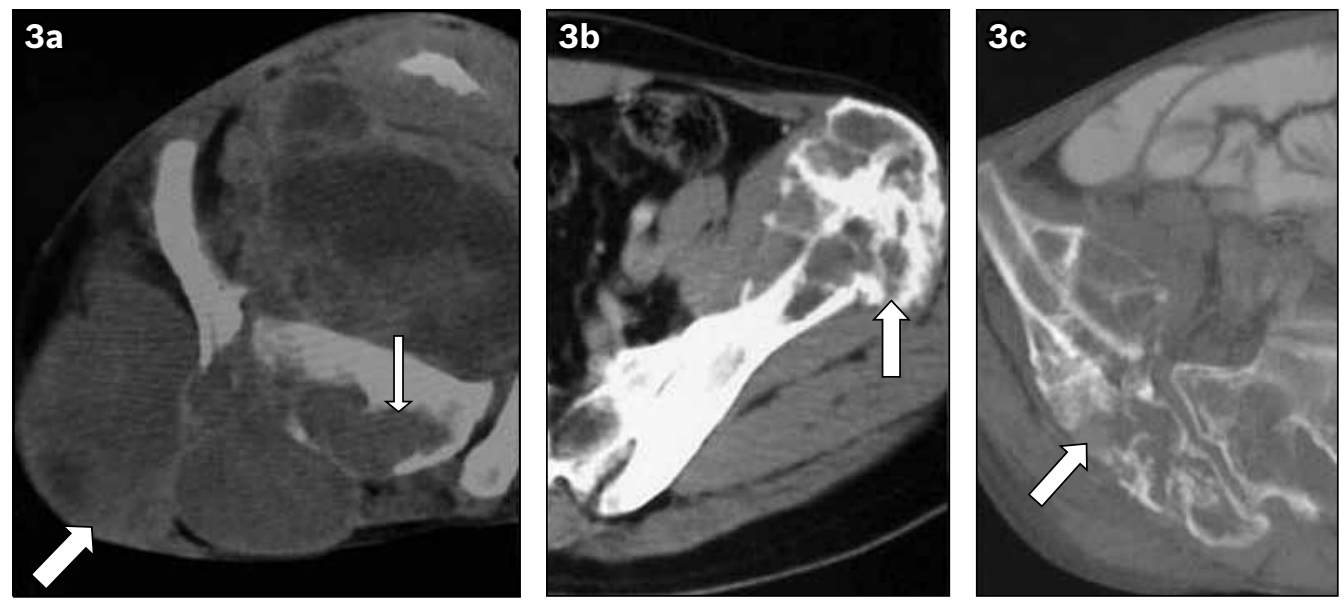

Fig. 3 Pelvic PNET. (a) Axial CT image of the right hemipelvis shows a large soft tissue mass with both intra- and extrapelvic components, with destruction of the sacrum (thick arrow) and extension into the spinal canal (thin arrow). (b \& c) Axial CT images of the pelvis show the healing phase of pelvic PNETs. The lack of significant soft tissue with mixed lytic-permeative change in the bone (thick arrows) suggests the healing nature of these lesions.

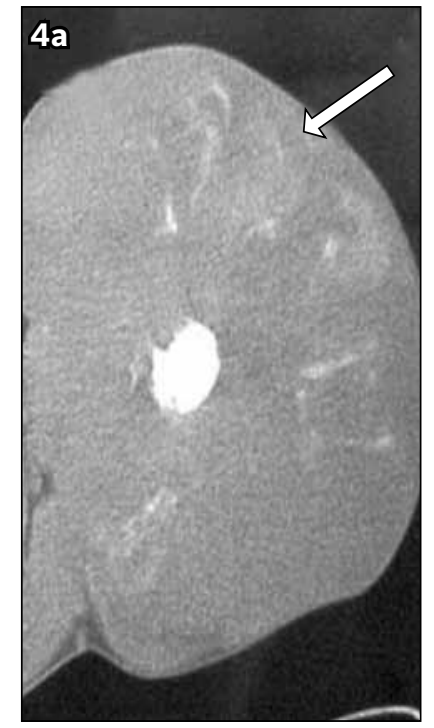

Fig. 4 Upper extremity PNET. (a) Axial CT image shows a large soft tissue mass encasing the left humerus, with destruction of the cortex and spiculated periosteal reaction (arrow). (b) Axial CT image shows a healing lesion involving the right humerus. The soft tissue mass is not seen, and the underlying bone shows sclerotic change overlapping the lytic destruction (arrow).
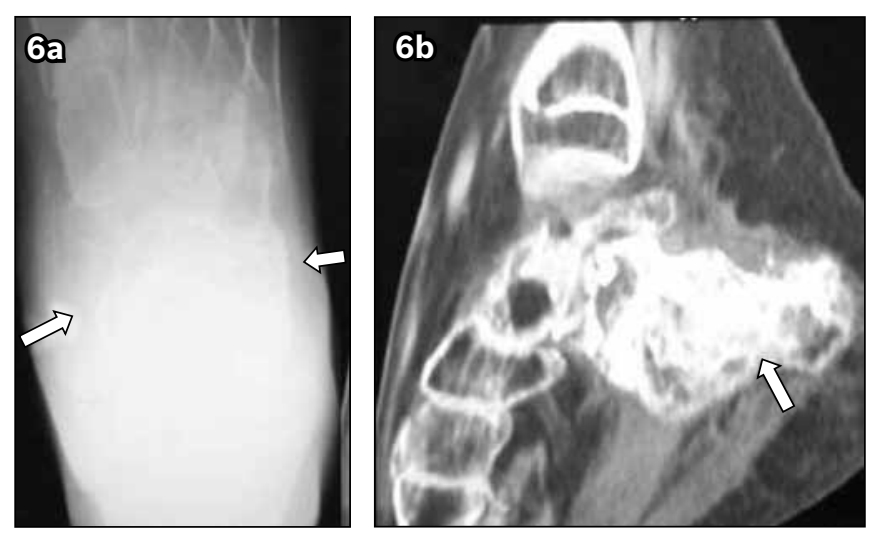

Fig. 6 Calcaneal PNET. (a) Radiograph of the right foot shows a poorly defined soft tissue mass (arrows) around the posterior part of the foot; bone erosions are not clearly seen on this radiograph. (b) Sagittal reformatted CT image shows destruction of the calcaneum (arrow), and the adjacent soft tissue.
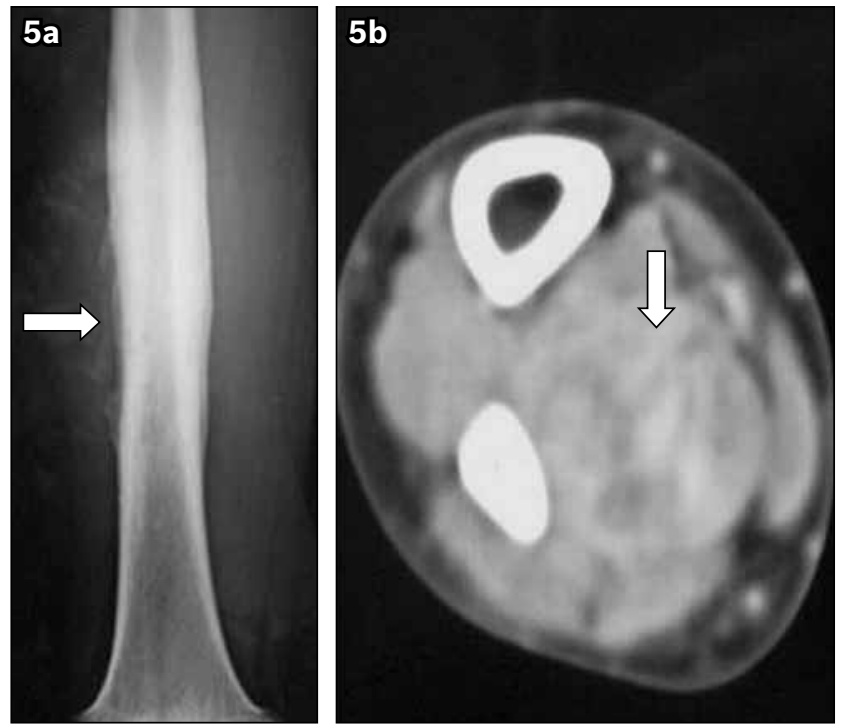

Fig. 5 Lower limb PNET. (a) Radiograph of the right femur shows permeative destruction of the shaft, with spiculated periosteal reaction (arrow) and adjacent soft tissue mass. (b) Contrast enhanced CT image of a different patient shows a heterogeneous mass in the posterior compartment muscles (arrow) of the tibia, with erosions and permeative cortical destruction (not shown).
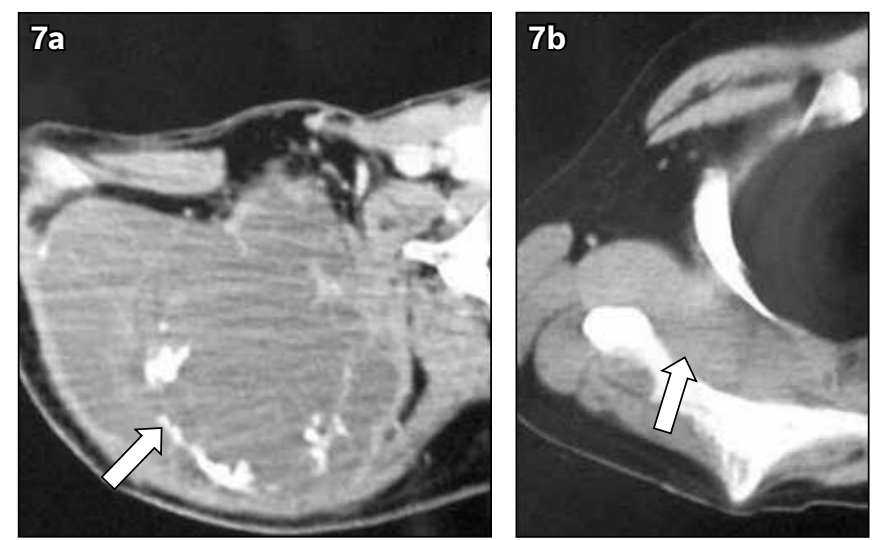

Fig. 7 PNET of the scapula in two different patients. (a) Axial CT image shows a large soft tissue mass with peripheral foci of calcification (arrow) noted in the region of the right scapula, with gross expansion and destruction of the scapula. The mass shows a large necrotic area. (b) CT image in a different patient shows a relatively smaller tumour (arrow) with less extensive destruction of the right scapula. 

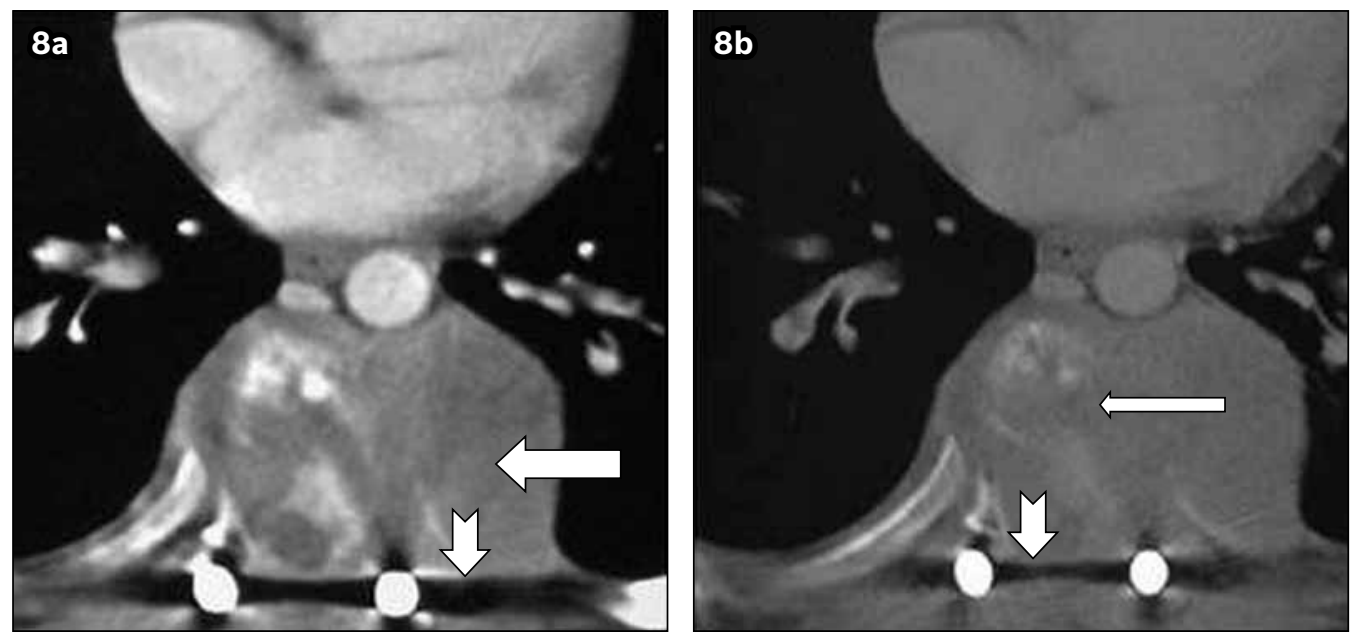

Fig. 8 Spinal PNET. Axial contrast-enhanced CT images in (a) mediastinal and (b) bone window settings at the level of T8 show a left paraspinal mass (thick arrow) causing destruction of the adjacent vertebral body (thin arrow). Streak artifacts (notched arrows) from fixation screws make the assessment of intraspinal extension difficult.
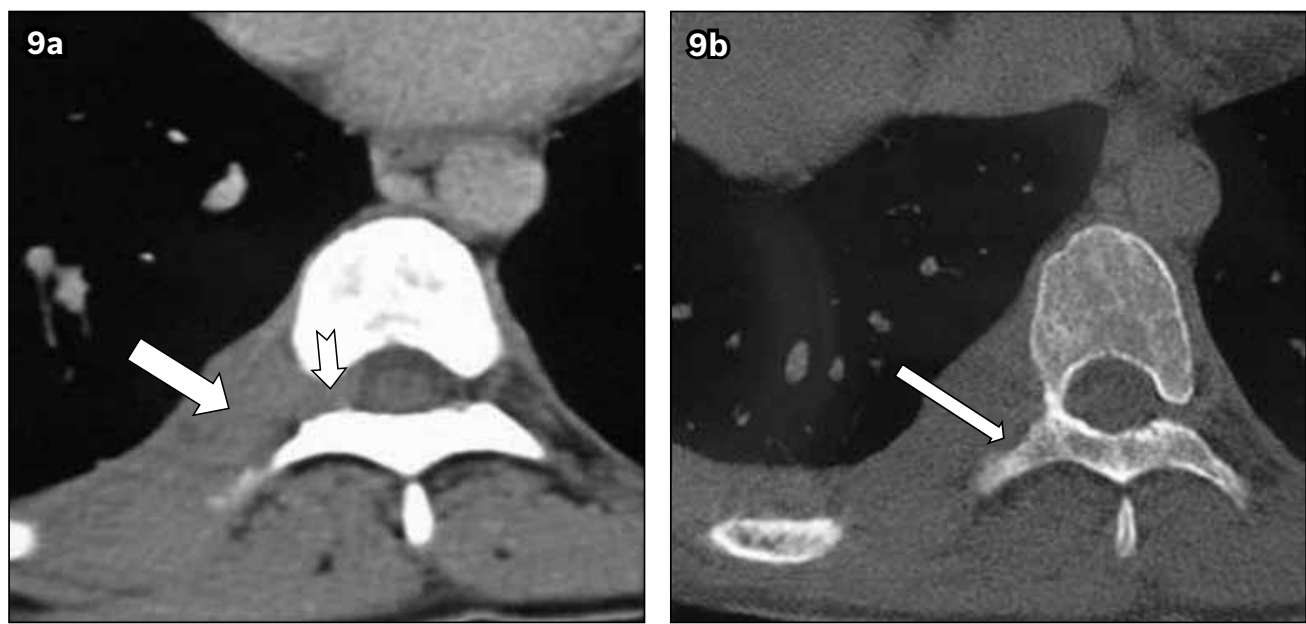

Fig. 9 Spinal PNET in another patient. Axial contrast-enhanced CT images in (a) mediastinal and (b) bone window settings at the level of T8 show a right paraspinal mass (thick arrow) causing erosion of the transverse process (thin arrow) and extension into the neural foramen (notched arrow). Although no extradural component is observed, note that MR imaging would be better able to demostrate intraspinal extension.
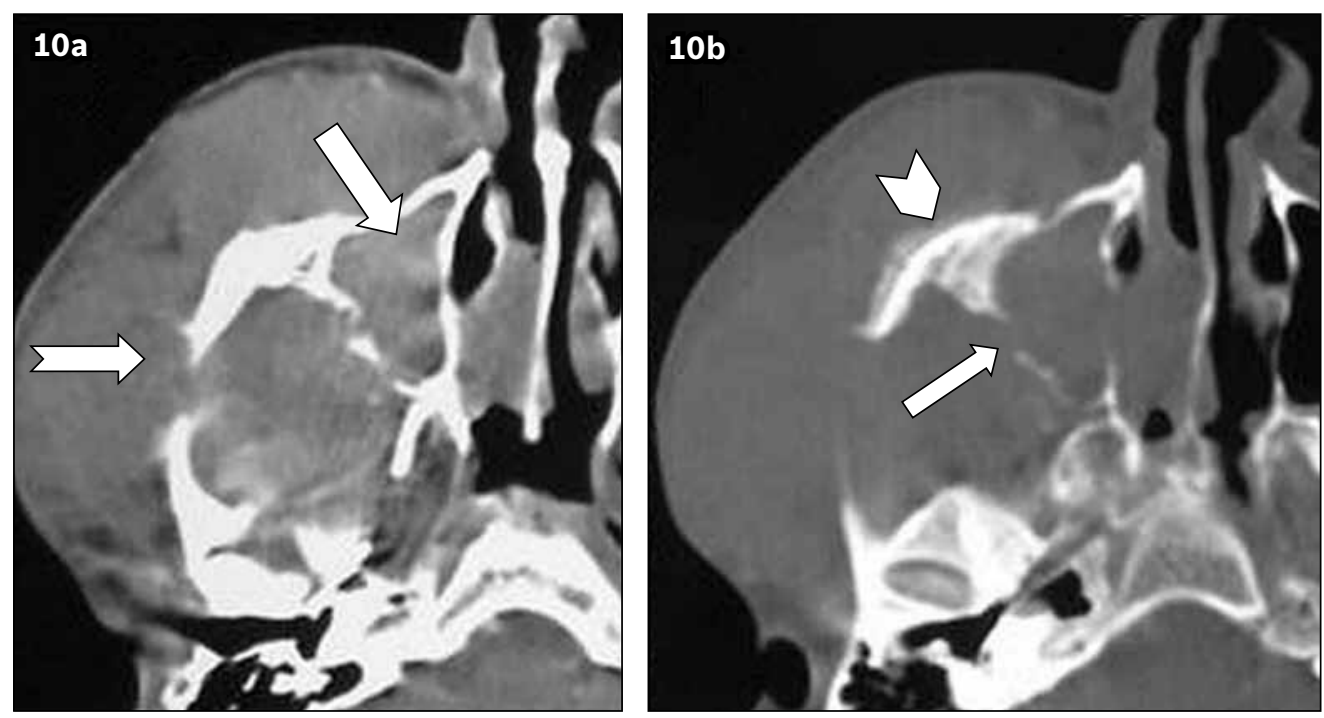

Fig. 10 PNET of the maxilla. Axial contrast-enhanced CT images of the maxillary sinuses in (a) soft tissue and (b) bone window settings show a large right sinonasal mass with a large component filling the right maxillary sinus (thick arrow), causing erosion of the posterior wall with retromaxillary component (thin arrow) and tumour extension into the infratemporal fossa and soft tissue of the cheek (notched arrow). Note the sclerosis and spiculated periosteal reaction involving the anterior wall of the maxillary antrum (arrowhead). 


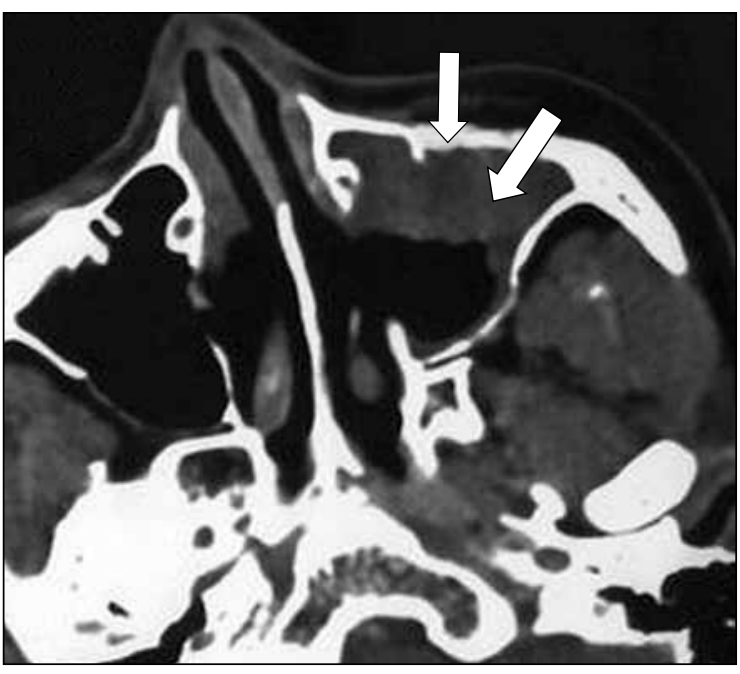

Fig. 11 PNET of the maxilla in another patient. Contrast-enhanced CT image shows a mass located predominantly along the inner aspect of the anterior wall of the left maxillary sinus (thick arrow), with erosion and remodelling of the wall (thin arrow). This tumour is much more limited than that shown in Fig. 10.

\section{Spine}

PNETs located in the spine lead to a clinical presentation of back pain. On imaging, a paraspinal soft tissue mass with destruction of the adjacent vertebra and intraspinal extension can be seen (Figs. 8 \& 9). MR imaging is useful for demonstrating the intraspinal component of the tumour. Like scapular PNETs, those originating from the spine also have a better prognosis. ${ }^{(10)}$

\section{Head and neck}

pPNETs can originate from the maxilla (Figs. 10 \& 11), mandible, ethmoid (Fig. 12), orbit or infratemporal fossa. These tumours tend to extend into various spaces, and intracranial extension is not uncommon. Orbital PNETs present with proptosis and visual impairment. On imaging, a retro-ocular infiltrative mass is typically noted (Fig. 13). The mandible is a rare site for PNETs, with only six cases reported so far; ${ }^{(12)}$ in three of these cases, the symphysis menti was the site of involvement. The general features of PNETs of the head and neck are similar to those located elsewhere. Differential diagnoses include metastasis, osteosarcoma, lymphoma, osteomyelitis and ameloblastoma. Neuroblastoma, a common primary tumour for metastasis in the early part of the first decade of life, frequently leads to multifocal lesions that may be indistinguishable from individual lesions of pPNET; multiplicity favours neuroblastoma metastases, as does calcification, when present. Osteosarcomas most frequently involve the long bones in children and young adults, and the craniofacial skeleton in middle-aged or elderly patients. Additionally, the presence of an ossified matrix associated with an aggressive lytic lesion favours the diagnosis of osteosarcoma. The head and neck region is a common site for primary lymphoma. In the absence of lymphadenopathy, the imaging appearance of lymphoma can be almost identical to that of pPNET. In such cases, diagnosis can only be made
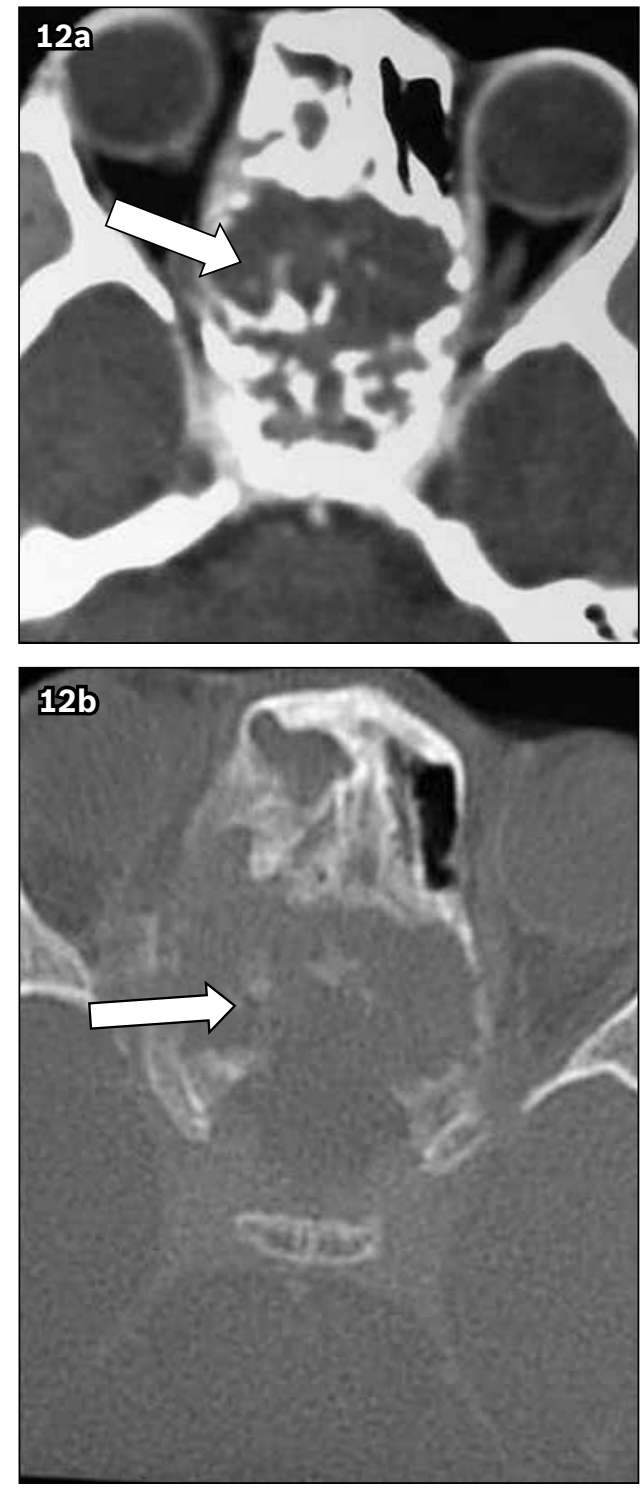

Fig. 12 PNET of the ethmoid bone. ( $a$ \& b) Contrast-enhanced CT images of the face at the level of the orbits show a large sinonasal mass (thick arrow) involving bilateral anterior and posterior ethmoid sinuses, causing extensive bone remodelling (thin arrow). There is no gross invasion of orbital fat.
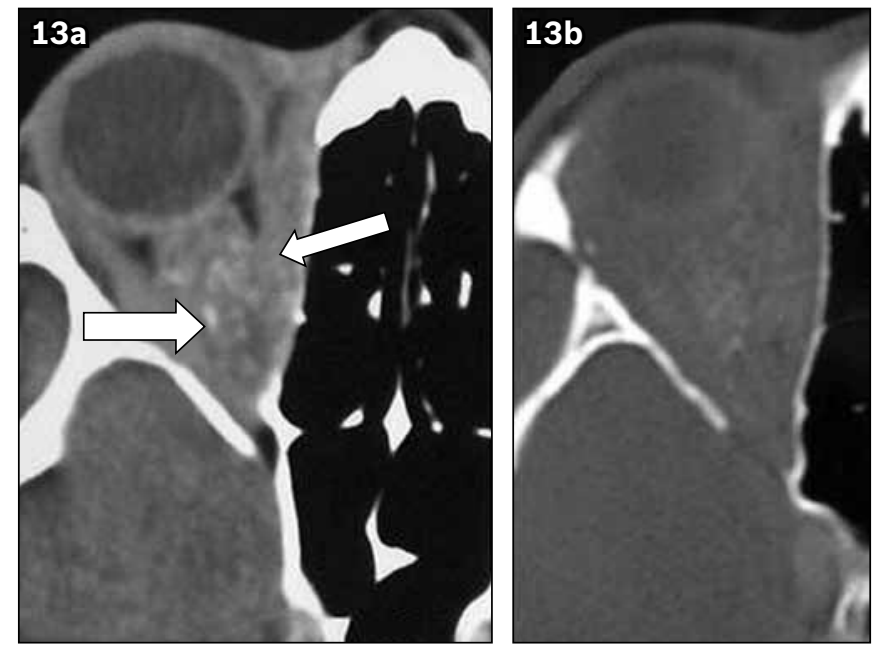

Fig. 13 Orbital PNET. (a \& b) Axial contrast-enhanced CT images of the orbit show a right retro-orbital mass with fine, speckled calcification (thick arrow). The medial rectus muscle is involved (thin arrow). There is no extension into the middle cranial fossa and no gross bone destruction. 

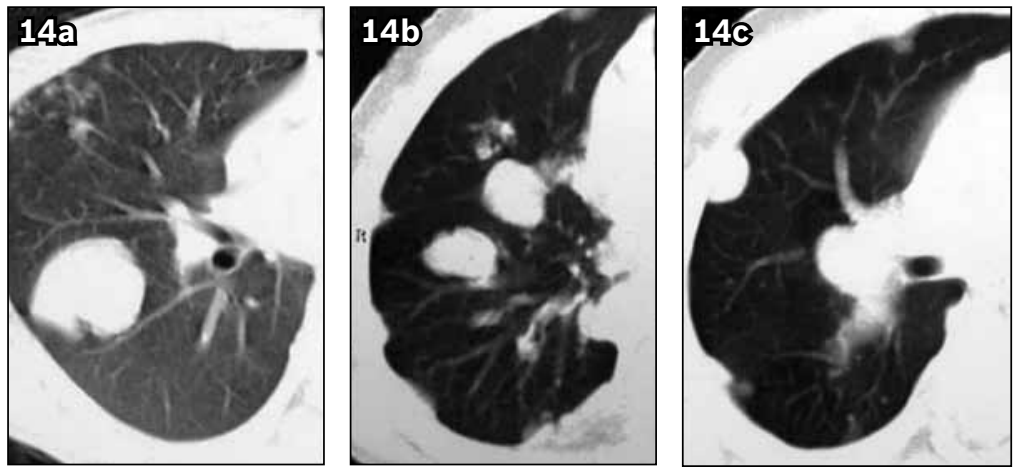

Fig. 14 PNET metastases to the lung and pleura. (a-c) Axial chest CT images in the lung window setting shows nodules in the lungs of patients with primary PNETs at various locations. The lung is the most common site of metastasis. Multiple pleural-based nodules in the right lung (Fig. 14c) are suggestive of pleural metastases in PNET.
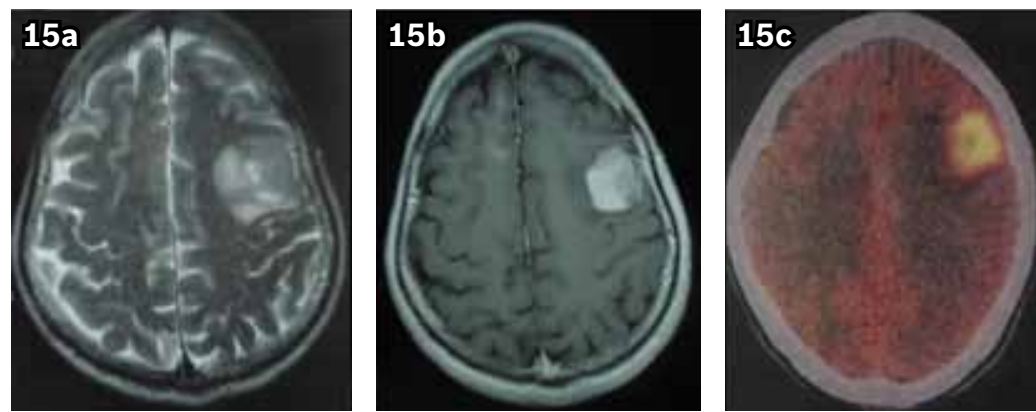

Fig. 15 PNET metastases to the brain. (a) Axial T2-W MR image of the brain of a patient with peripheral PNET of the right femur shows a well-defined, isointense to hyperintense mass in the left posterior frontal cortex and subcortical white matter with surrounding hyperintensity suggestive of oedema. (b) T1-W MR image obtained after administration of intravenous gadolinium shows marked homogeneous contrast enhancement. (c) Axial PET-CT image shows marked uptake in the lesion.

at biopsy. Osteomyelitis can be reliably differentiated based on the patient's clinical history, as well as the imaging findings of sequestra and sinuses. Ameloblastoma has a decided predilection for the body of the mandible, and the orientation and relatively well-defined borders of this lesion help to distinguish it from pPNET.

\section{METASTASES OF PERIPHERAL PNET}

The most common sites of metastases are the lungs (Fig. 14), pleura (Fig. 15), bones, bone marrow, liver and brain (Fig. 16). Chest CT is frequently done at presentation and follow-up in patients with metastatic pPNET. Although the incidence of metastases varies according to the primary site of the tumour, metastasis is a fairly common occurrence.

\section{CONCLUSION}

pPNET can be considered in the differential diagnosis of soft tissue tumour presenting in various locations of the body. Features that favour a diagnosis of PNET over lymphoma, neuroblastoma, rhabdomyosarcoma or carcinoma include large heterogeneous soft tissue mass, adjacent bone destruction or erosion, displacement rather than encasement of structures, lymphadenopathy and absence of calcification. ES belongs to the same family of tumours as PNET and both have similar imaging features, although ES lacks neural differentiation, which is characteristic of PNETs.

\section{REFERENCES}

1. Dehner LP. Peripheral and central primitive neuroectodermal tumors. A nosologic concept seeking a consensus. Arch Pathol Lab Med 1986; 110:997-1005.

2. Rosai J. Soft tissues. In: Rosai and Ackerman's Surgical Pathology. St. Louis: Mosby, 2004: 2324-5.

3. Khong PL, Chan GC, Shek TW, Tam PK, Chan FL. Imaging of peripheral PNET: common and uncommon locations. Clin Radiol 2002; 57:272-7.

4. Isotalo PA, Agbi C, Davidson B, et al. Primary primitive neuroectodermal tumor of the cauda equina. Hum Pathol 2000; 31:999-1001.

5. Dorfmuller G, Wurtz FG, Umschaden HW, Kleinert R, Ambros PF. Intraspinal primitive neuroectodermal tumour: report of two cases and review of the literature. Acta Neurochir (Wien) 1999; 141:1169-75.

6. Sallustio G, Pirronti T, Lasorella A, et al. Diagnostic imaging of primitive neuroectodermal tumour of the chest wall (Askin tumour). Pediatr Radiol 1998; 28:697-702.

7. Ibarburen C, Haberman JJ, Zerhouni EA. Peripheral primitive neuroectodermal tumors: CT and MRI evaluation. Eur J Radiol 1996; 21:225-32.

8. Kolb EA, Kushner BH, Gorlick R, et al. Long-term event-free survival after intensive chemotherapy for Ewing's family of tumors in children and young adults. J Clin Oncol 2003; 21:3423-30.

9. Hari S, Jain TP, Thulkar S, Bakhshi S. Imaging features of peripheral primitive neuroectodermal tumours. Br J Radiol 2008; 81:975-83.

10. Dick EA, Mchugh K, Kimber C, Michalski A. Imaging of noncentral nervous system primitive neuroectodermal tumors: diagnostic features and correlation with outcome. Clin Radiol 2001; 56:206-15

11. Kim MS, Kim B, Park CS, et al. Radiologic findings of peripheral primitive neuroectodermal tumor arising in the retroperitoneum. AJR Am J Roentgenol 2006; 186:1125-32.

12. Yeh $\mathrm{CH}$, Yeow KM, Chu SY et al. Imaging findings in mandibular primitive neuroectodermal tumour: a report of a rare case and review of the literature. Dentomaxillofac Radiol 2011; 40:451-6. 


\section{SINGAPORE MEDICAL COUNCIL CATEGORY 3B CME PROGRAMME} (Code SMJ 201308B)

Question 1. The following is an immunohistochemical marker of primitive neuroectodermal tumours (PNETs):
(a) CD 34.
(b) CD 19.
(c) CD 99.
(d) CD 117.

Question 2. PNETs typically occur in:
(a) Children.
(b) Adolescents and young adults.
(c) The elderly.
(d) Neonates.

Question 3. The following is the most common site of occurrence of PNETs:
(a) Chest wall.
(b) Paraspinal location.
(c) Pelvis.
(d) Head and neck.

Question 4. Regarding the treatment of PNETs:
(a) Radiotherapy is curative.
(b) Chemotherapy is curative.
(c) Surgery with adjuvant chemotherapy and radiotherapy is the preferred choice.
(d) Surgery alone is uniformly curative.

Question 5. Regarding the prognosis for PNETs:
(a) Excellent with a high five-year survival rate.
(b) Treatment results in complete cure.
(c) Dismal despite aggressive therapy.
(d) Recurrences are rare.

\footnotetext{
Doctor's particulars:

Name in full

MCR number

Specialty:

Email address

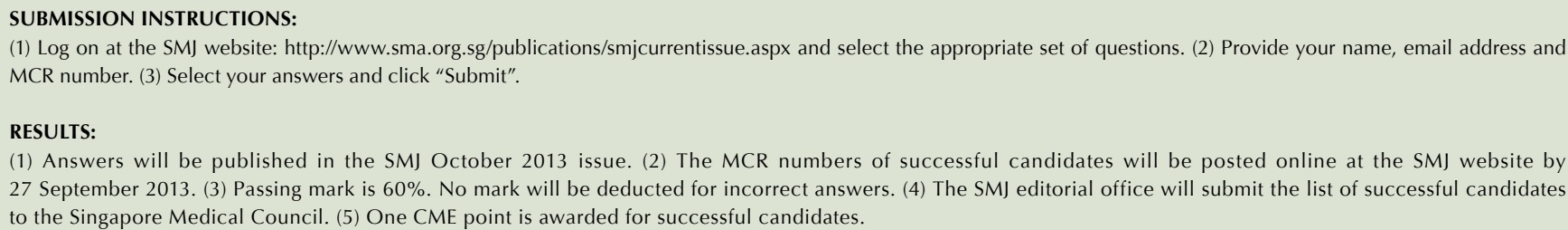
MCR number. (3) Select your answers and click "Submit".

RESULTS:

(1) Answers will be published in the SMJ October 2013 issue. (2) The MCR numbers of successful candidates will be posted online at the SMJ website by 27 September 2013. (3) Passing mark is $60 \%$. No mark will be deducted for incorrect answers. (4) The SMJ editorial office will submit the list of successful candidates to the Singapore Medical Council. (5) One CME point is awarded for successful candidates.

(1) Log on at the SMJ website: http://www.sma.org.sg/publications/smjcurrentissue.aspx and select the appropriate set of questions. (2) Provide your name, email address and

Deadline for submission: (August 2013 SMJ 3B CME programme): 12 noon, 20 September 2013.
} 